\title{
Hipofosfatemia revertida al extirpar hemangioendotelioma compuesto del dedo mayor del pie
}

Jorge Sapunar $Z^{1}$, Juan Carlos Roa $\mathbf{S}^{2}$, Susana Moscoso A*. Reversion of hypophosphatemia after the excision of a composite hemangioendothelioma in a great toe. Report of one case

We report a 43 years old male admitted to the hospital for progressive lumbar pain, lasting 20 years, that caused severe disability. On admission the patient had a serum phosphate of $2 \mathrm{mg} / \mathrm{dl}$, an urine phosphate excretion over $300 \mathrm{mg} / \mathrm{dl}$ and serum alkaline phosphatases over $750 \mathrm{U} / \mathrm{L}$ Serum intact parathormone was normal and tubular maximum phosphorus/glomerular filtration was $0.7 \mathrm{mg} / \mathrm{dl}$. Bone scintigraphy showed an increased radionuclide uptake in condro-costal joints. Bone densitometry showed femoral osteoporosis. A violet colored mass was detected in a great toe. It was removed and the pathological diagnosis was a composite hemangioendothelioma. After tumor excision, serum phosphate levels returned to normal values and symptoms disappeared within 15 days (Rev Méd Chile 2003; 131: 909-14).

(Key Words: Hemangioendothelioma; Hypophosphatemia; Phosphoric acid esters)

Recibido el 2 de enero, 2003. Aceptado en versión corregida el 12 de junio, 2003.
${ }^{1}$ Unidad de Endocrinología, Facultad de Medicina, Universidad de La Frontera.
2Servicio de Anatomía Patológica, Hospital Regional de Temuco.
*Residente de Medicina Interna, Hospital Regional de Temuco.

L a hipofosfatemia es un hallazgo relativamente frecuente en pacientes hospitalizados, pero que excepcionalmente tiene relevancia clínica. La hipofosfatemia puede ser el resultado de pérdidas renales, reducción en la absorción intestinal 0 redistribución del fosfato al interior de la célula. Las pérdidas renales de fosfato explican la mayor parte de las hipofosfatemias y, a su vez, pueden ser consecuencia de defectos intrínsecos de la función tubular, de cambios en la volemia, del efecto de drogas o de anomalías en la secreción de hormo-

Correspondencia a: Dr. Jorge Sapunar Z. Antumalal 01024, Temuco. Fono-fax: 45-735778. E-mail: finfin@surnet.cl jsapunar@ufro.cl nas que regulan la reabsorción tubular de fosfato ${ }^{1}$.

A propósito de un caso de hipofosfatemia asociada a un tumor de origen vascular, se discuten recientes avances en la comprensión de la homeostasis del fosfato.

\section{Caso CLínICO}

Varón de 43 años, agricultor de etnia mapuche, residente en área rural próxima a Chol-Chol en la Cordillera de la Costa de la provincia de Cautín, que consultó, en julio de 2000 en el policlínico de Reumatología del Hospital Regional de Temuco, por dolor lumbar recurrente de 20 años de evolución, no asociado con traumatismo ni con actividad física, sin irradiación y tratado habitualmente con paracetamol 


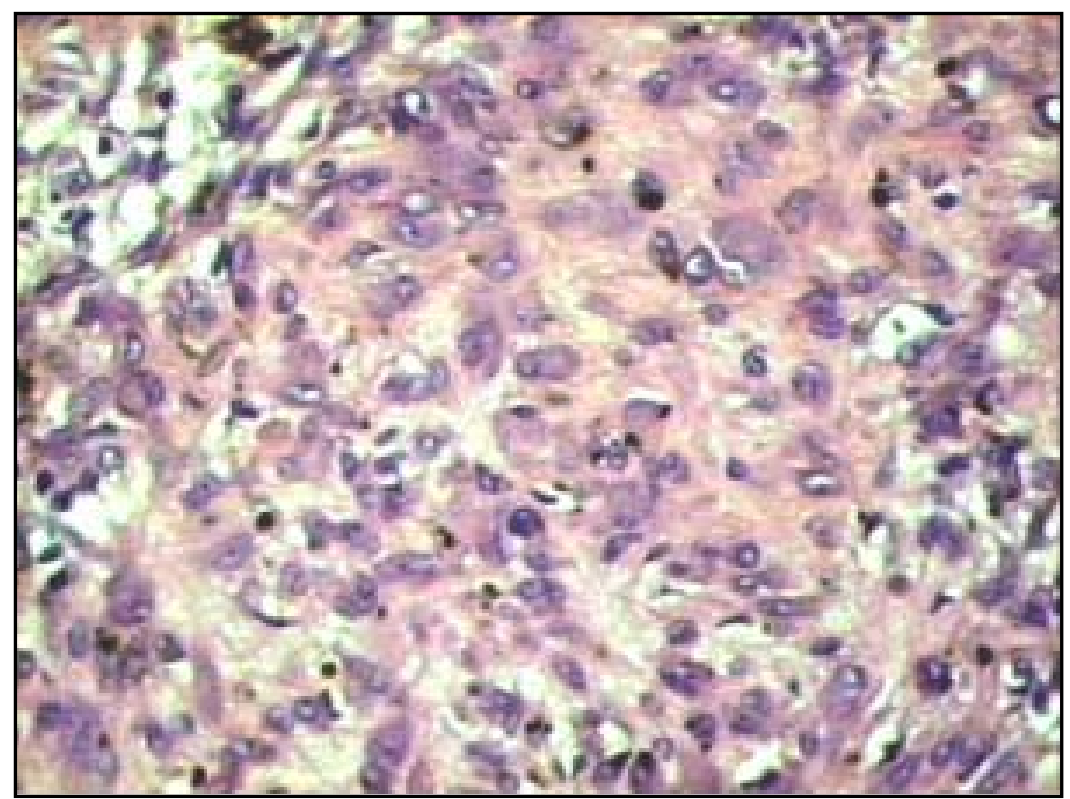

Figura 2. Microscopia.

A. Microfotografía Hematoxilina Eosina 200x. Se reconoce componente epiteloideo.

B. Microfotografía Hematoxilina Eosina 100x. Se reconoce componente fusocelular con depósito de hemosiderina.

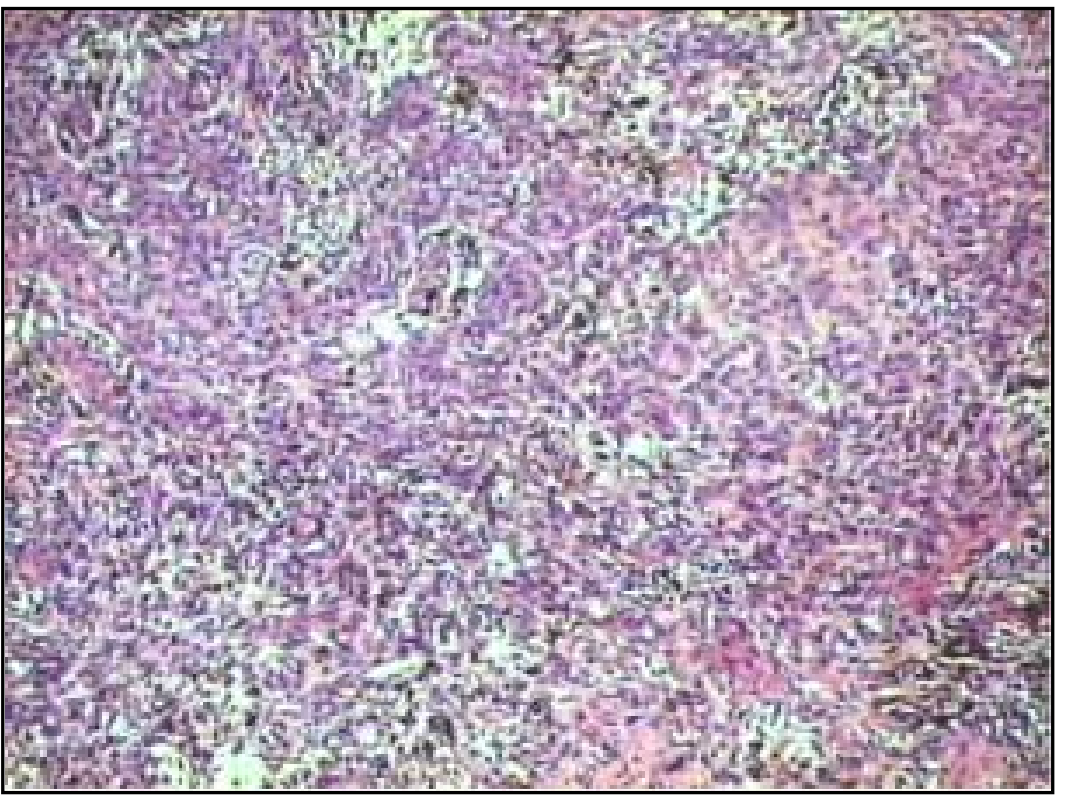

y 2. El diagnóstico fue hemangioendotelioma compuesto.

Luego de $72 \mathrm{~h}$ sin mediar tratamiento específico para la hipofosfatemia se comprobó la remisión de ésta. En una semana el dolor lumbar y la impotencia funcional habían desaparecido, el paciente fue dado de alta con jarabe de fosfato y vitamina D, siendo citado a control para evaluar radioterapia complementaria.

\section{Discusión}

La asociación entre un tumor de partes blandas, hipofosfatemia y osteomalacia fue reconocida por primera vez en 1947 por McCance. En 1964, Dent 


\section{Tabla 2. D iagnósticos histológicos en osteomalacia oncogénica}

\author{
Hemangiopericitoma \\ Hemangioma esclerosante \\ Tumor mesenquimático osificante \\ Osteoblastoma \\ Hemangioma cavernoso \\ Granuloma reparativo de células gigantes \\ Tumor maligno de células gigantes \\ Fibroma no osificante \\ Tumor como masa de osteoide degenerado \\ Neurinoma maligno \\ Carcinoma de células pequeñas \\ Adenocarcinoma de próstata
}

*En orden de frecuencia.

y Friedman demostraron que la resección del tumor normalizaba la fosfatemia ${ }^{2,3}$.

Hasta ahora se han comunicado algo más de 100 casos de osteomalacia oncogénica cuyos elementos esenciales son hipofosfatemia, hiperfosfaturia, normocalcemia, niveles normales de 25 $\mathrm{OH}$ D3 y disminuidos de 1-25 OH D3, concentraciones séricas de PTH y péptido relacionado con PTH normales y ausencia de otros defectos en la reabsorción tubular ${ }^{1-14}$. En la Tabla 2 se mencionan los tipos histológicos más frecuentes y en la Tabla 3 sus localizaciones.

Actualmente se acepta que la osteomalacia oncogénica se debe a la producción tumoral de uno o más factores que inhiben la reabsorción tubular de fosfato y la hidroxilación en posición 1 aaa de la vitamina $\mathrm{D}^{15}$. El hasta ahora desconocido factor fosfatúrico, denominado fosfatonina, inhibe el co-transporte de sodio y fosfato en cultivos de células tubulares renales de diferentes mamíferos, sin utilizar el receptor de PTH ni activar el sistema adenilciclasa ${ }^{16}$. El medio de cultivo condicionado con el tumor, como se mencionó anteriormente, también inhibe la actividad de la enzima $1 \alpha$ hidroxilasa de las células

Tabla 3. Localizaciones tumorales en osteomalacia oncogénica

Tumores extraóseos

- Ortejo mayor

- Región maleolar

- Rodillas

- Muslos

- Axilas

- Faringe y laringe

- Pared abdominal

Tumores óseos

- Todo el fémur

- Costillas

- Radio y cúbito distal

- Etmoides

- Ileon

- Húmero

- Mandíbula renales, sin que se sepa a la fecha si este efecto depende de la fosfatonina u otra sustancia ${ }^{1}$.

Se han identificado una gran variedad de proteínas elaboradas por tumores asociados a hipofosfatemia, destacando FGF-23 y FRP-4 como posibles factores fosfatúricos ${ }^{17-19}$

Los pacientes con raquitismo hipofosfatémico ligado al cromosoma X tienen un fenotipo bioquímico extremadamente semejante a la osteomalacia oncogénica, sugiriendo que ambas enfermedades pudieran compartir mecanismos patogénicos.

Los pacientes con raquitismo hipofosfatémico ligado al cromosoma X tienen mutaciones en el gen PHEX, que sorprendentemente no codifica moléculas involucradas en el transporte de fosfato, sino que se asemeja a genes que codifican endopeptidasas ${ }^{20}$. Estos antecedentes han llevado a postular que la proteína codificada por PHEX degradaría fosfatoninas, que normalmente actuarían en el riñón produciendo fosfaturia. En osteomalacia oncogénica la producción excesiva de fosfatonina ¿FGF-23 ó FRP-4?, sobrepasaría la acción de la proteína codificada por PHEX, en cambio en el raquitismo hipofosfatémico la ausencia o disminución de actividad de ésta se asociaría 
con un aumento en los niveles circulantes de fosfatina ${ }^{1}$.

El hemangioendotelioma compuesto es una entidad recientemente descrita que caracteriza a un grupo de neoplasias de origen vascular en las que se reconoce una mezcla de patrones morfológicos que varían considerablemente en cada caso, alternando elementos histológicos de benignidad, bajo y alto grado de malignidad, lo que explica su alto índice de recidiva local. Existen 2 comunicaciones que reúnen 9 casos y que coinciden en lesiones que se originan en la hipodermis de las manos y pies, evolucionando por años. Todos fueron diagnosticados en individuos adultos, con un promedio de edad de 40 años en una serie y

\section{REFERENCIAS}

1. Kumar R. Tumor induced osteomalacia and regulation of phosphate homeostasis. Bone 2000; 27: 333-8.

2. Weiss D, Bar RS, Weidner N, Tener M, Lee F. Oncogenic osteomalacia: strange tumours in strange places. Postgrad Med J 1985; 61: 349-55.

3. Mc Clure J, Smith PS. Oncogenic osteomalacia. J Clin Pathol 1987; 40: 446-53.

4. Parker MS, Kiein I, Haussler MR, Mintz DH. Tumor Induced Osteomalacia. JAMA 1981; 245: 492-2????

5. RYAN EA, ReISS E. Oncogenous osteomalacia. Review of world literature of 42 cases and report of two new cases. Am J Med 1984; 77: 501.

6. TAYLOR HC, FaLION MD, Velasco ME. Oncogenic Osteomalacia and Inappropiate Antidiuretic Hormone Secretion due to Oat Cell Carcinoma. Ann Intern Med 1984; 101: 786-8.

7. McGuire MH, Merenda JT, Etzkorn JR, Sundaram M. Oncogenic Osteomalacia. Clin Orthop 1987; 244: 305-8.

8. van Heyningen C, Green ART, MacFarlane IA, BuRRow CT. Oncogenic hypophosphataemia and ectopic corticotrophin secretion due to oat cell carcinoma of the trachea. J Clin Pathol 1994; 47: un caso único de 23 años en la otra. La recurrencia se observó en 3 de los 9 pacientes, uno de ellos con metástasis ganglionar linfática regional. En ninguno de los casos se describe una osteomalacia oncogénica asociada ${ }^{21,22}$.

Nuestro caso cumple con la mayor parte de los criterios para una osteomalacia oncogénica. Lamentablemente no disponíamos de las determinaciones de $25 \mathrm{OH}$ y 1-25 OH D3; sin embargo, la PTHi normal y la normocalcemia descartaban un hiperparatiroidismo primario. La magnitud de la fosfaturia en presencia de hipofosfatemia y la resolución de los síntomas y trastornos bioquímicos luego de la resección del tumor sugieren fuertemente el diagnóstico propuesto.

80-2.

9. Schapira D, Izhak OB, Nachtigal A, Burstein A, Shalom RB, Shagrawi I Et al. Tumor Induced Osteomalacia. Sem Arthrit Rheum 1995; 25: 3546.

10. Avila NA, Skarulis M, Rubino DM, Doppman JL. Oncogenic Osteomalacia: Lesion detection by MR Skeletal Survey. AJR 1996; 167: 343-5.

11. David K, Revesz T, Kratimenos G, Krausz T, CROCKARD HA. Oncogenic osteomalacia associated with a meningeal phosphaturic mesenchymal tumor. J Neurosurg 1996; 84: 288-92.

12. REESE DM, Rosen PJ. Oncogenic osteomalacia associated with prostate cancer. J Urology 1997; 158: 887.

13. Wilkins GE, Granleese S, Hegele RG, Holden J, ANDERSON DW, Bondy GP. Oncogenic Osteomalacia: Evidence for a Humoral Phosphaturic Factor. J Clin Endocrinol Metab 1995; 80: 1628-34.

14. Clunie GPR, Fox PE, Stamp TCB. Acquired Hypophosphatemic Oncogenic Osteomalacia: Problems of diagnosis, treatment and long-term management. Bone 2001; 28 (5 Suppl): S120.

15. Cai Q, Hodgson SF, Kao PC, Lennon VA, Kiee GG, ZINSMIESTER AR ET AL. Inhibition of renal phosphate transport by a tumor product in a patient with oncogenic osteomalacia. N Engl J Med 1994; 330: 1645-9. 
16. Popovtzer MM. Tumor induced hypophosphatemic osteomalacia (TUO): evidence for a phosphaturic cyclic AMP-independent action of tumor extract. Clin Res 1981; 29: 418A.

17. Bowe AE, Finnegan R, Jan de Beur SM, Cho J, Levine MA, KUMAR R ET AL. FGF-23 inhibits renal tubular phosphate transport and is PHEX substrate. Biochem Biophys Res Commun 2001; 284: 977-81.

18. KUMAR R. New insights into phosphate homeostasis: fibroblast growth factor 23 and frizzledrelated protein- 4 are phosphaturic factors derived from tumors associated with osteomalacia. Curr Opin Nephrol Hypertens 2002; 11: 547-53.

19. Larsson T, Zahradnik R, Lavigne J, Ljuggren O,
JuPPNER H, JONSSON KB. Immunohistochemical detection of FGF-23 protein in tumors that cause oncogenic osteomalacia. Eur J Endocrinol 2003; 148: 269-76.

20. DREZNER MK. PHEX gene and hyphosphatemia. Kidney Int 2000; 57: 9-18.

21. Naylen SJ, Rubin BP, Calonje E, Chan JK, Fletcher CD. Composite hemangioendothelioma: A complex, low-grade vascular lesion mimicking angiosarcoma. Am J Surg Pathol 2000; 24: 352-61.

22. ReIs-FilHo JS, PaIva ME, Lopes JM. Congenital composite hemangioendothelioma: Case report and reppraisal of the hemangioendothelioma spectrum. J Cutan Pathol 2002; 29: 226-31. 